\title{
Téoros
}

Revue de recherche en tourisme

\section{Le service à la clientèle}

Pour réussir la qualité de service et satisfaire la clientèle touristique

\section{Laurent Bourdeau}

Volume 17, numéro 3, automne 1998

Pratiques contemporaines de gestion dans le domaine du tourisme

URI : https://id.erudit.org/iderudit/1072149ar

DOI : https://doi.org/10.7202/1072149ar

Aller au sommaire du numéro

Éditeur(s)

Université du Québec à Montréal

ISSN

0712-8657 (imprimé)

1923-2705 (numérique)

Découvrir la revue

Citer cet article

Bourdeau, L. (1998). Le service à la clientèle : pour réussir la qualité de service et satisfaire la clientèle touristique. Téoros, 17(3), 5-10.

https://doi.org/10.7202/1072149ar d'utilisation que vous pouvez consulter en ligne.

https://apropos.erudit.org/fr/usagers/politique-dutilisation/ 


\title{
A N A L Y S E
}

\section{Le Service À LA CLIENTÈLe}

\author{
POUR RÉUSSIR LA QUALITÉ DE SERVICE \\ ET SATISFAIRE LA CLIENTĖLE TOURISTIQUE
}

\section{Laurent Bourdeau}

Traditionnellement, Lorsqu'il est question du service à la clientelè d'une entreprise, nombre de gestionnaires et de clients se reportent au traitement des plaintes et au retour des marchandises. Une telle perception du service à la clientèle, conforme à la réalité pendant plusieurs années, ne correspond plus aux exigences du milieu commercial. En effet, aujourd'hui, en plus de bien gérer ses plaintes, l'entreprise doit également eviter de susciter l'insatisfaction de sa clientele.

En tourisme, comme dans tous les secteurs de service, l'objectif premier de l'entreprise n'est pas d'offrir le meilleur système de gestion des plaintes, mais de satisfaire le client. Comment l'entreprise touristique peut-elle satisfaire sa clientèle ? C'est ce que nous démontrerons dans cet article ; plus précisément, nous verrons comment le service à la clientèle peut devenir l'instrument pour une entreprise touristique qui garantira la qualité du service et, par conséquent, la satisfaction de la clientèle.

Afin de comprendre le rôle du service à la clientèle, nous nous attarderons à l'ambivalence qui existe entre l'hétérogénéité et la standardisation du service. Par la suite, nous verrons la place qu'occupe la participation du touriste dans la production du service, ainsi que la relation entre l'entreprise et le client. De plus, nous nous attarderons à la relation entre la qualité et les normes de service, deux concepts permettant au service à la clientèle de satisfaire le client. Afin de situer la qualité de service dans un contexte plus global, nous porterons une attention particulière à l'expérience du touriste et à la commercialisation des produits touristiques au niveau international, ainsi qu'au rôle de l'État dans la qualité et les normes de service. Finalement, nous tenterons de voir comment le service à la clientèle peut devenir le canal par lequel les gestionnaires. traitent la relation avec les touristes.

\section{L'AMBIVALENCE HÉTÉROGÉNÉITÉ / STANDARDISATION}

Le contenu du produit touristique que doivent gérer les entreprises est avant tout intangible. Cette caractéristique, spécifique au secteur des services, pose un défi de taille aux gestionnaires : il est impossible de contrôler toutes les composantes du produit touristique. C'est en tenant compte de cette contrainte que les gestionnaires devront satisfaire le touriste. En effet, si certaines variables sont contrôlables, telles que le nombre d'employés ou le budget de publicité, d'autres variables ne le sont pas, notamment la météo, l'humeur ou les besoins de la clientèle. Ce constat soulève le problème de l'hétérogénéité du service. En d'autres mots, il est difficile de reproduire un service de façon identique sans en altérer la qualité. Le gestionnaire se trouve alors devant un défi important : comment assurer une qualité de service qui soit constamment satisfaisante pour le client ? La réponse la plus simple réside dans la standardisation du service.
Cependant, cette solution n'apparaît que partielle car la gestion du service touristique doit tenir compte de l'ambivalence entre hétérogénéité et standardisation. D'un côté, les gestionnaires cherchent à standardiser le service dans le but de contrôler les effets négatifs liếs à une trop grande hétérogénéité de l'expérience touristique. D'un autre côté, les gestionnaires ne peuvent offrir un service complètement standardisé ou trop rigide, car ils détruiraient l'essence même du tourisme. En effet, pourquoi visiter un endroit particulier si l'on trouve la même chose ailleurs? Pourquoi voyager si le service est tellement standardisé qu'il n'y a plus de contact humain ? La standardisation complète du service peut ainsi avoir des conséquences négatives sur l'attitude des touristes. Évidemment, le degré de standardisation dépend du type de service touristique offert. Certains services parviennent à standardiser - dans une certaine mesure - avec succès leur processus de production ; pensons à McDonald's. Dans plusieurs pays, ce type de restaurant peut constituer une référence pour le touriste étranger qui a peu d'expérience de voyage, en réduisant son insécurité et son dépaysement. Le touriste se trouve alors devant un point d'ancrage familier et peut investir davantage d'énergie affective et cognitive dans d'autres éléments dépaysants du voyage.

En fait, pour le gestionnaire la difficulté réside à trouver un équilibre entre l'hétérogénéité et la standardisation du service. En d'autres mots, il s'agit de trouver un equilibre entre un service personnalisé et un service standardisé, entre un produit touristique sur mesure et un produit touristique identique pour l'ensemble de la 
clientèle, entre des tâches plus ou moins définies et des tâches routinières pour le personnel. L'équilibre ne réside pas nécessairement à mi-chemin entre l'hétérogénéité et la standardisation du service touristique. Tout dépend du type de service que veut offrir l'entreprise. Chose certaine, le positionnement du service n'apparaît pas se situer à une des deux extrémités de ce continuum, soit à cause des coûts occasionnés par une trop grande hétérogénéité du service, soit à cause de l'absence de personnalisation due à une trop grande standardisation du service. C'est donc en tenant compte de cette ambivalence entre le caractère hétérogène du service et sa standardisation, que le gestionnaire devra élaborer les dimensions de la qualité du service à offrit.

\section{LA PRODUCTION DU SERVICE ET LA RELATION AVEC LE CLIENT}

Si la participation du touriste à la production du service, ou à la servuction ${ }^{I}$, est essentielle à la production du service, sa présence est également source d'hétérogéneité. Par contre, pour les gestionnaires, la présence du touriste est synonyme de variable incontrôlable. En fait, c'est la combinaison entre la participation du client à la production du service — faible par opposition à élevée — et l'intangibilité du service - intangible par opposition à tangible -, qui détermine le degré de contrôle du gestionnaire sur la servuction.

S'il est difficile de reproduire un service de la même façon chaque fois qu'un client consomme l'expérience touristique, on peut s' interroger sur la possibilité d'assurer un service de qualité chaque fois que la prestation est offerte. Bien que les gestionnaires contrôlent dans une certaine mesure la qualité de la prestation offerte à l'aide du personnel en contact, ils ne contrôlent pas directement le comportement du touriste, qui est un des principaux acteurs de la prestation de service. En fait, peu d'entreprises touristiques se sont intéressées à la gestion des deux acteurs de la production du service, soit le touriste et le personnel en contact. Pour régler ce problème, certaines entreprises se sont tournées vers un marketing touristique. Bien que le marketing soit défini comme étant une philosophie de gestion des organisations qui reconnaît l'importance de la satisfac- tion de la clientèle ${ }^{2}$, force est de constater que la gestion de la relation entre l'entreprise et le touriste ne reçoit pas toujours l'attention qu'elle mérite. D'ailleurs, comme le constate l'État québécois, * Le client n'est pas suffisamment au cour des préoccupations de l'industrie touristique québécoise ${ }^{3}$. En fait, en n'accordant guère d'attention à la relation client-entreprise, le gestionnaire risque de n'avoir aucune influence sur la satisfaction du client.

Pour bien gérer la relation client-entreprise dans le secteur des services, certaines entreprises mettent sur pied un service à la clientèle et nomment un responsable du service à la clientèle. Une des principales tâches de ce service est alors de gérer la qualité du service. Cela apparaît d'autant plus pertinent si l'on tient compte du fait que la satisfaction du client est fortement liée à la qualité du service ${ }^{4}$.

\section{LA QUALITÉ ET LES NORMES DE SERVICES}

Afin de satisfaire la clientèle, les gestionnaires d'une entreprise touristique doivent donc définir ce qu'ils désirent comme qualité de service ; pour ce faire, ils peuvent se tourner vers les modèles de qualité de service existants dans le secteur des services.

Bien qu'il existe quelques recherches portant sur la qualité du service offert dans différents secteurs touristiques, notamment dans les agences de voyages ${ }^{5}$, il n'existe pas à proprement parler de modèle type de la qualitế de service pour le tourisme. Ajoutons que les études portant sur la qualité des services dans le secteur touristique découlent principalement du modèle de Parasuraman et coll. ${ }^{6}$ et de Berry et coll. ${ }^{7}$. Fortement utilisé en marketing, ce modèle définit la qualité de service selon cinq grandes dimensions. La première, l'assurance, se traduit par l'habileté du personnel à établir une relation de confiance avec le client. La compétence et la courtoisie du personnel sont des elements déterminants pour établir une relation de confiance. La qualité du service s'appuie notamment sur le fait que le personnel des entreprises touristiques connailt le contenu de l'offre et qu'il est également capable de le communiquer aux touristes. Par exemple, un guide touristique doit connaî= tre la région qu'il fait visiter aux points de vue géographique, historique, culturel et économique, mais il doit également être en mesure de transmettre ses connaissances et son intérêt aux touristes.

La deuxième dimension de la qualité de service, l'empathie, suppose que le personnel de l'entreprise touristique est en mesure d'accorder une attention particulière à ses clients tout en pouvant pressentir ses attentes. Cette dimension apparait fort pertinente si l'on considère que les attentes des touristes ne sont pas toujours exprimées avec clarté. En effet, les attentes du touriste sont souvent vagues et floues ${ }^{8}$; en conséquence, il ne les exprime pas toujours facilement. Il est alors difficile pour les gestionnaires et le personnel de comprendre ses besoins". En fait, comme les gestionnaires peuvent difficilement mesurer les attentes des touristes, l'entreprise doit faire appel à la capacité du personnel à pressentir les attentes, les motivations et les valeurs de sa clientèle.

La troisième dimension, la fidélité, s'exprime par l'habileté de l'entreprise à livrer avec précision le service promis. Cette dimension permet de porter une attention particulière à l'ambivalence hétérogénéité - standardisation. La décision stratégique sera prise en fonction du positionnement de l'entreprise, mais peu importe le niveau de standardisation, l'entreprise devra assurer à ses clients un minimum d'uniformité par la servuction. En d'autres mots, toute entreprise devra être en mesure de reproduire les dimensions clés de son service et ce, à chaque prestation.

La quatrième dimension de la qualité de service, la disponibilité, porte sur la capacité et la volonté exprimées par le personnel d'aider les consommateurs avec promptitude. Bien que le temps de réaction d'un service varie selon le type de prestation, le touriste désire être traité promptement ou, du moins, sentir qu'on s'occupe de lui. Il est important de démontrer que le processus de production du service commence aussitôt que le client utilise le service. Dans le cas des lignes d'attentes d'une compagnie aérienne, le client devra avoir l'impression que le service débute dès son arrivée, par exemple, un agent lui confirmera qu'il est bien dans la bonne file d'attente.

La cinquième dimension, la tangibilité, se rapporte aux équipements physiques, à l'apparence du personnel, à la communi- 
cation et au prix ; c'est-à-dire à tout ce qui permet de rendre tangible le service. Le gestionnaire doit alors chercher à préciser le type de service qu'il désire offrir à la clientèle. $\mathrm{C}^{7}$ est en rendant le service tangible que le gestionnaire parviendra à diminuer les attentes vagues et floues des touristes. Cette dimension apparaît fort importante à la prise de décision du touriste avant l'achat d'un produit touristique.

De façon à rendre les cinq dimensions de la qualité de service opérationnelles dans l'entreprise, le gestionnaire peut utiliser des normes de service (voir figure 1). Bref, les normes de service représentent l'outil permettant d'assurer la qualité de service grâce à la relation client-entreprise et d'encadrer l'expérience de consommation touristique.

Les normes de service découlent de la définition de la qualité de service établie par l'entreprise et visent à régulariser les comportements des deux acteurs : le personnel en contact et le client ${ }^{16}$. Cet encadrement se fait alors par une définition du rôle êt des tâches du personnel en contact et du touriste par le processus de servuction. Bien entendu, moins le service est tangible, plus le personnel et le touriste seront encadrés. Pour le personnel en contact, les normes de service prennent la forme de directives leur attribuant un pouvoir de décision et une définition de tâches conformes aux dimensions de la qualité de service élaborées par l'entreprise. Pour le touriste, les normes de service prennent la forme d'un encadrement facilitant la consommation de l'expérience de service. Par exemple, elles peuvent se traduire par des panneaux de signalisation visant à diriger les flux de circulation dans un musée.

En plus d'encadrer le personnel et la clientèle par le processus de servuction, les normes de service deviennent des promesses aux yeux de la clientèle ${ }^{11}$. Les normes de service, affichées à l'intéricur de l'agence de voyages ou présentées dans une publicité, rendront le service tangible tout en devenant des normes de qualité de service. Par exemple, une compagnie ferroviaire pourrait établir comme norme : * nos trains partent et arrivent toujours à l'heure s, mais elle devra s'assurer de pouvoir réaliser cette promesse.

En fait, les normes de service prennent la forme d'énoncés visant à régulariser les

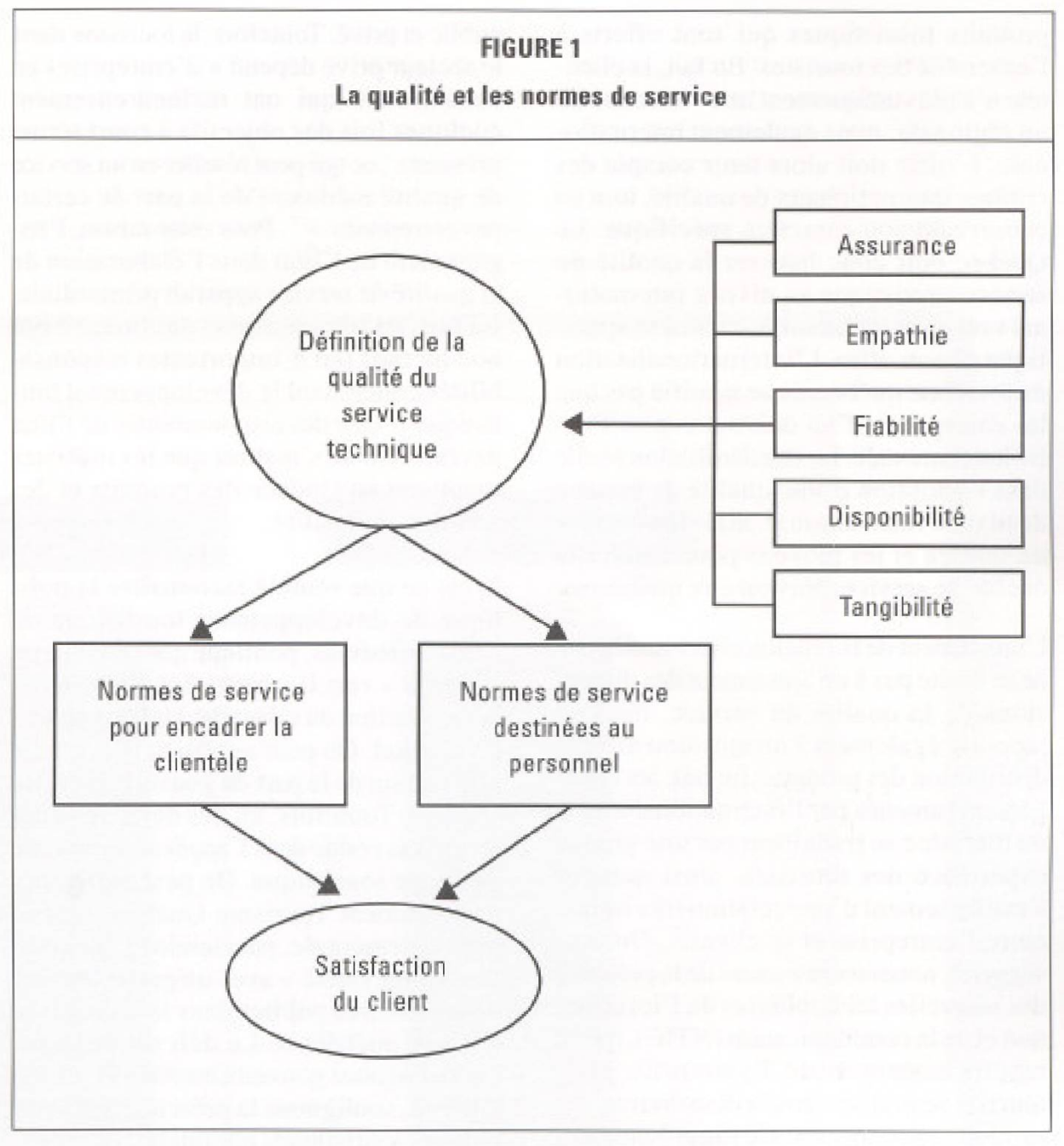

comportements des acteurs de la production d'un service touristique, soit le personnel et le touriste. Sans tomber dans la standardisation complète, les normes de service permettent une certaine souplesse dans la production du service. En d'autres mots, elles permettent aux gestionnaires $\mathrm{d}^{4}$ assurer la qualite de service tout en tenant compte de l'ambivalence hêtétrogénéité / standardisation $^{12}$.

C'est grâce à l'utilisation de ces normes que le gestionnaire parviendra à offrir un service de qualité tenant compte des caractéristiques spécifiques de l'expérience touristique, telles que le caractère hédoniste, intangible et hétérogène de ce service. Les normes de service ne sont pas une panacée permettant de prévoir tous les comportements du personnel en contact et du touriste, mais représentent le moyen ou un guide que l'entreprise utilise pour assurer la qualité du service nécessaire à la satisfaction du client.

\section{L'EXPÉRIENCE DU TOURISTE ET LA COMMERCIALISATION INTERNATIONALE}

L'internationalisation des voyages et l'augmentation du nombre de voyages au Québec ont permis aux touristes d'accroittre leur expérience. Les touristes sont davantage expérimentés et exigeants ${ }^{13}$, en ce qui concerne les voyages, mais également la prise de décision ${ }^{14}$. En conséquence, les touristes comparent de plus en plus la qualité des services qu"ils reçoivent dans les entreprises touristiques et sont de plus en plus difficiles à satisfaire. On peut d'ailleurs suggérer que le touriste évalue la qualité de service en comparant le service reçu de l'entreprise québécoise avec les autres services expérimentés lors de voyages au Québec, mais également au Canada et à l'étranger.

Dans son désir de satisfaire les clients, l'entreprise locale doit tenir compte des 
produits touristiques qui sont offerts à l'ensemble des touristes. En fait, la clientèle n'a plus uniquement une vision locale ou régionale, mais également internationale. L'offre doit alors tenir compte des critères internationaux de qualité, tout en conservant son caractère spécifique. Le Québec doit donc hausser la qualité du service touristique au niveau international tout en maintenant le caractère spécifique de son offre. L'internationalisation du tourisme québécois ne signifie pas que les entreprises d'ici doivent copier l'offre internationale. La standardisation réside dans l'adoption d'une qualité de service de niveau international, mais les normes de service et les moyens pour assurer la qualité de service, doivent être québécois.

L'ajustement de la relation client-entreprise ne se limite pas an ajustement des dimensions de la qualité du service, mais se rapporte également à un ajustement de la distribution des produits. En fait, les changements amenés par l'internationalisation du tourisme se traduisent par une grande experience des touristes, ainsi que par l'établissement d'une relation très intime entre l'entreprise et le client $^{15}$. On peut suggérer, notamment à cause de la présence des nouvelles technologies de l'information et de la communication (NTIC), qu'un rapprochement entre l'entreprise et le touriste se réalise dans la distribution des produits touristiques. Ce rapprochement pourrait toutefois se traduire par une diminution du contact humain. On peut penser que l'utilisation des NTIC réduira le nombre d'agences de voyages ayant pignon sur rue car les NTIC ouvrent la voie à une distribution directe des produits touristiques entre le producteur et le client. A moyen terme, la distribution des produits touristiques risque d'être chambardée par la présence du commerce électronique qui pourrait conduire à la mise sur pied d'un marché électronique mondial pour les produits touristiques $^{16}$. Encore une fois, cette caractéristique de l'internationalisation indique que les dimensions de la qualité de service doivent être de calibre international. Pensons au fait que le touriste utilisant Internet, confortablement installe à son bureau ou à la maison, peut choisir entre plusieurs destinations et comparer la qualite des offres touristiques.

\section{LE RÔLE DE L'ÉTAT}

La gestion et le développement touristiques sont la responsabilité des secteurs public et privé. Toutefois, le tourisme dans le secteur privé dépend a d'entreprises en concurrence qui ont malheureusement quelques fois des objectifs à court terme pressants; ce qui peut résulter en un service de qualité médiocre de la part de certailnes entreprises $s^{17}$. Pour cette raison, l'engagement de l'État dans l'élaboration de la qualité de service apparaît primordiale. En fait, les organisations publiques à but non lucratif ont d'importantes responsabilités concernant le développement touristique $^{18}$. Une des responsabilités de l'État devrait être de s'assurer que les touristes reçoivent au Québec des produits et des services de qualité.

C"est ce que semble reconnaître la politique de développement touristique de l'Etat québécois, politique qui annonce un * virage $n$ vers le service à la clientèle où la satisfaction du client devient un objectif primordial. On peut se réjouir d'une telle affirmation de la part du gouvernement du Québec. Toutefois, un des défis de l'État québécois réside dans l'application de cette politique touristique. On peut se demander comment Tourisme Québec, agence gouvernementale, parviendra à instaurer le $\alpha$ virage client $x$ avec un grand nombre de partenaires publics dans le milieu touristique québécois. Le défi est de taille. Outre l'agence gouvernementale Tourisme Québec, soulignons la présence des associations touristiques régionales, des MRC et de plusieurs associations sectorielles intervenant sur le plan touristique. La présence d'autant de joueurs accroit les divergences dans la façon de concevoir la qualité de service. À ce sujet, mentionnons que l'État québécois reconnaît le manque de concertation entre les différents partenaires de l'industrie touristique, notamment, entre $\alpha$ les régions touristiques qui se voient encore souvent comme étant concurrentes plutôt que partenaires ; la concertation entre les associations touristiques régionales et sectorielles est trop faible $x^{19}$.

En plus des difficultés associées à la définition de la qualité de service, l'État québécois « ne dispose pas d'un système integré lui permettant de cerner les attentes et le comportement de ces clientèles, ainsi que leur degré de satisfaction, à l'égard de leur séjour au Québec (produits offerts. accueil, qualité des services) $x^{20}$. Cette absence de système intégré apparaît comme une des raisons de l'insuceès du marketing touristique ${ }^{21}$. En d'autres mots, non seulement l'État québécois n'est pas encore en mesure de définir ce qu'est la qualité du service dans le tourisme québécois, mais sa mesure à l'échelle nationale ne peut être réalisée. Dans un tel contexte, l'objectif marketing de la satisfaction du client ne peut être atteint par des actions ciblées et mesurables.

La solution, comme le propose le gouvernement du Québec, passe par une reconnaissance de Tourisme Québec comme ctant $\alpha$ le chef de file en tourisme $*^{n}$. Ce chef de file du tourisme québécois devra intégrer une même vision de la qualité de service chez tous les partenaires de l'industrie touristique et ce, afin de définir ce que doit être la qualité de service du produit touristique québécois ${ }^{23}$. De leur côté, les entreprises touristiques devront élaborer leurs propres dimensions de la qualité de service en lien avec la vision intégrée de l'État québécois. Bien qu'il puisse y avoir des dimensions communes, telles que celles que nous avons vues précédemment et qui furent proposées par Parasuraman et coll. ${ }^{24}$, chaque entreprise touristique québécoise devrait élaborer ses propres dimensions de la qualité de service.

Face à I'internationalisation du tourisme et a l'accroissement de la concurrence, les entreprises touristiques québécoises doivent done rehausser la qualité des services touristiques offerts. Comme nous $l^{\prime}$ 'avons vu, dans un premier temps, l'atteinte de cet objectif passe par l'engagement de l'État dans l'élaboration et la mesure de la qualité de service au niveau national. Dans un deuxième temps, les entreprises touristiques doivent emprunter le a virage client $*$ et assurer la qualité du service. En fait, toute entreprise qui désire entreprendre le $\alpha$ virage client $w$ doit s'inscrire dans une perspective de marketing relationnel où un service de qualité devient une condition sine qua non de satisfaction des diverses clientèles touristiques.

\section{LE SERVICE À LA CLIENTÈLE : LE CANAL POUR LA GESTION DE LA RELATION}

Au cours des dernières années, les entreprises touristiques québécoises ont développé de nouveaux produits touristiques et sont parvenues à les vendre tant au niveau local qu'à l'échelle internationale. La prochaine étape de la commercialisation 
des produits touristiques québécois exige des gestionnaires l'établissement d'un canal privilégié avec le client et ce, dans le but de satisfaire ses besoins adéquatement. Si l'État québécois peut jouer un rổle moteur dans la concertation, il ne peut remplacer les entrepreneurs qui sont en contact direct avec la clientèle.

C'est grâce à l'implantation d' un service, ou à la désignation d'un responsable du service à la clientèle, que l'entreprise parviendra à appliquer les normes de service qui permettent de contrôler et d'accroître la qualité du service. C'est donc par' l'intermédiaire du service à la clientèle, qui est en quelque sorte la courroie de transmission entre le client et l'entreprise, que se gère la relation client-entreprise. Dans cette perspective, le client est au cour de la relation.

Sous la responsabilité du département de marketing, le service à la clientèle d'une entreprise appliquera les politiques marketing et cherchera, par l'établissement de normes de service, à encadrer le personnel en contact et le client. Le service a la clientèle, ou son responsable, permettront à l'entreprise de définir et de mesurer la qualité du service, mais aussi d'élaborer et d'appliquer les normes de service. Bref, ce service est $l^{\prime}$ outil qui permet de gérer la relation entreprise-touriste afin de satisfaire le touriste. La mise sur pied d'un tel service représente un premier pas vers le $x$ virage client $x$.

La gestion d'une expérience touristique plutôt que d'un produit touristique demeure une des principales conditions de réussite d'un service à la clientèle de qualité. Cette vision de l'offre commerciale conduit les gestionnaires à vouloir établir une relation avec le client. Selon cette vision, l'entreprise n "offre plus une vente et un produit, mais cherche a offrit un service pour l'ensemble de la relation entre le client et l'entreprise. Ce changement dans les actions marketing de l'entreprise a pour but de satisfaire le client, mais également de le fidéliser. L'établissement d'un canal a done pour but de satisfaire le client pour qu"il revienne visiter le Québec ou qu'il recommande la destination à son entourage. La fidélisation de la clientèle est fort importante si l'on considère que le degré de fidélisation est intimement relié avec la perception de la qualité du service.

\section{CONCLUSION}

Dans cet article, nous avons vu que les changements dans le milieu commercial obligent les entreprises touristiques à revoir leurs actions marketing. L' internationalisation du tourisme, tant au niveau de la distribution que de la concurrence, ainsi que l'expérience développée par les touristes, influencent les exigences de la clientèle en matière de produits touristiques. Ces changements exigent que les entreprises touristiques québécoises établissent des normes de service de niveau international qui tiennent compte du caractère spécifique du Québec, mais éga-

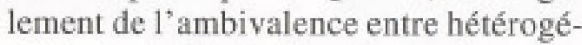
néité et standardisation du service.

Afin de réaliser la qualitế du service, 1'État québécois et les entreprises touristiques doivent se concerter et assumer des responsabilités partagées. Au niveau gouvernemental, Tourisme Québec est l'agence qui doit assumer ce rôle, tandis qu'au niveau des entreprises touristiques, c'est le service à la clientèle ou son responsable qui doit assurer le a virage client $w$. II appartient donc a chaque entreprise touristique de définir la qualité de service qu'elle désire offrir et, surtout, de définit les moyens pour appliquer et hausser sa qualité de service grâce aux normes de service. Cependant, l'État québécois se doit de mesurer la qualité des services dans chacun des secteurs de $1^{\prime}$ industrie touristique afin de veiller à ce que le Québec offre un service qui satisfasse les normes internationales. En conséquencc, l'État doit proposer, dans un premier temps, des dimensions de la qualité de service puis, dans un deuxième temps, chercher à accroître la qualité du service offert par les entreprises touristiques québécoises.

L'objectif premier de l'entreprise réside dans l'atteinte d'une qualité de service qui puisse satisfaire la clientèle de façon a la fidéliser. Le service à la clientèle, ou son responsable, peut faciliter l'atteinte de cet objectif en devenant $\mathrm{l}^{4}$ instrument par lequel une entreprise touristique établit une relation avec la clientèle. En d'autres mots, le responsable du service à la clientèle doit connaître les attentes du client, définir et mesurer la qualité de service, ainsi que développer les outils et les normes de service permettant de satisfaire la clientèle.
Aujourd'hui, en raison des changements du milieu commercial et de $\mathrm{l}^{\text {t'avenement }}$ du marketing des services, les gestionnaires doivent comprendre l'importance de gérer l'ensemble de la relation cliententreprise. C'est à partir du service à la clientèle que peut se réaliser la gestion de cette relation. Ce service devient le canal de la relation entre les deux acteurs de la prestation du service, soit le touriste et le personnel en contact.

En résumé, le service à la clientèle s'assurera de définir la qualité du service et utilisera des normes de service afin de satisfaire les touristes. Le service à la clientèle, ou son responsable, doit toujours avoir à l'esprit que la qualité et la satisfaction du service sont deux concepts différents, mais fortement interreliés. C'est autour de ces deux concepts que la gestion de la relation entre l'entreprise et le client doit se faire.

Laurent Bourdeau est professeur de marketing au Département d'économie agroalimentaire er des sciences de la consommation de I'Universite Laval.

Un comité de lecture a lu et accepté ce texte

\section{NOTES}

1 Le terme servuction s, proposé par Eiglier et Langeard (1987), représente le processus de production du service et se compose des. termes * service * et * production *.

2 Filiatrault et Chebat, 1984.

3 Québec, 1998: 12.

4 Eiglier et Langeard, 1987 ; Nguyen, 1991.

5 ex. Fick et Ritchie, 1991 ; LeBlane, 1992 ; Ogden, 1998 ; McCutcheon, 1994 ; Ryan et Cliff, 1997.

6 Parasuraman et coll., 1985.

7 Berry et coll., 1990.

8 Jayanti et Jackson, 1991.

9 Ajoutons que le degré d'expérience du touriste face au service, ainsi que le degré d'intangibilitế de la prestation façonnent la prise de décision face à l'achat du produit touristique. Plus le touriste a d'expérience des voyages, moins le service est perçu comme étant intangible et plus la prise de décision 
pour l'achat d'un voyage est facile. On peut affimer que le touriste experimenté a davantage de facilité à exprimer ses attentes et que la täche des gestionnaires de tangibiliser le service touristique offert est ainsi facilitée.

10 Alain, 1998.

11 Alain, 1998.

12 Les normes doivent être suffisamment rigides afin de contrer les effets négatifs associés a l'hétérogénéité du service, mais doivent également laisser place a l'ajustement du service selon les attentes et les besoins spécifiques des différentes clientèles.

13 Vallerand, 1998.

14 Afin d'illustrer l'incidence de l'expérience touristique accumulée par les touristes au cours des dernières années, pensons à l'aug. mentation du nombre de critêres dans le processus de prise de décision du produit touristique. Le touriste québécois ou étranger en visite au Québec est souvent émerveille par la nature, la beauté des paysages, l'hospitalité et la culture québécoise. Cependant, dans le choix de sa destination ét dans l'élaboration de son sentiment de satisfaction, le touriste ne se limite plus à ces seuls critères. Pour prendre sa décision d'achat, le touriste tiendra compte des caractéristiques spécifiques de la destination touristique ou de l'image projetée par cette destination, mais il tiendra également compte du niveau de service qu'il pourra obtenir.

15 Vallerand, 1998.

16 La commereialisation des produits touristiques sur Internet semble déjà bien engagée si l'on observe le nombre de sites Web of frant la possibilite d'acheter un voyage directement sur l'autoroute virtuelle. Pensons aux compagnies aériennes qui ont dépassê le stade de la promotion sur Internet pour passer au stade de la commercialisation en utilisant la billetterie électronique.

17 Filiatrault et Chebat, $1984 ; 2$.

18 Filiatrault et Chebat, 1984.

19 Québec, $1998: 12$.

20 Québec, 1998: 12.

21 Filiatrault et Chebat. 1984.

22 Québec, 1998: 12. Mentionnons que, dans sa politique de développement, l'État québécois appelle à une redéfinition du partenariat entre Tourisme Québec et les ATR tout en reconnaissant ces dernières comme des partenaires privilégiés en région.
23 Pour s'assurer que les composantes de base de l'offre touristique québécoise respectent les normes internationales de qualité, l'État québécois se propose d'implanter * en partenariat avec l'industrie, un programme de qualite [...] A plus long terme, un sceau de qualité, qui pourrait être par exemple « Bonjour Québec s, viendra reconnaitre et promouvoir les entreprises touristiques qui auront adheré au programme * (Tourisme Québec, $1998: 16$ ).

24 Parasuraman et coll., 1985.

\section{RÉFÉRENCES}

Alain, Marcel (1998), Réussir la qualite de service, Montréal, Editions Nouvelles.

Berry, Leonard L., Valarie A. Zeithaml et A. Parasuraman, (1990), Five Imperatives for Improving Service Quality *, Sloan Managenent Joumal, vol. 51, été

Eiglier, Pierre, et Éric Langeard (1987). Servuction: le marketing des services, Paris, McGraw-Hill.

Fick, Gavin, et J.R. Brent Ritchie (1991), \& Measuring Service Quality in the Travel and Tourism Industry $s$, Journal of Travel Research, automne, p. 2-9.

Filiatrault, Pierre, et Jean-Charles Chebat (1984), \& Le marketing touristique au Québec, un essai diagnostic $\%$, Teoros, vol. $3, \mathrm{n}^{0} 1, \mathrm{p}, 2-4$.

Jayanti, Rama, et Anita Jackson (1991), * Service Satisfaction : An Exploratory Investigation of Three Models $n$, Advances in Consumer Research, sous la direction de Rebecca H. Holman et Micheal R. Solomon, Ann Arbor, MI, Association for Consumer Reseach, vol. 18, p. 603-610.

LeBlanc, Gaston (1992), \& Factors Affecting Customer Evaluation of Service Quality in Travel Agencies : An Investigation of Customer Perceptions *, Journal of Travel Research, printemps, p. 10-16.

McCutcheon, David M. (1994), \& Service Quality Planning in Urban Tourism : Tradeoffs and Resource Deployment $w_{\text {, Proceedings of the }}$ Quality Management in Urban Tourism: Balancing Business and Environment, sous la direction de Peter E. Murphy, p. 56-66.

Nguyen, Nha (1991), "Un modèle explicatif de l'évaluation de la qualité d'un service : une étude empirique w, Rechenche et Applications en Markering, vol, 6, $\mathrm{n}^{\circ} 2$, p. 83-97.

Ogden, Harold J. (1998), * Travel Agent Service Quality and the Leisure Traveller $\%$, Actes de I'Association canadienne des sciences de l"administration, Division gestion du tourisme et de I'accueil, sous la direction de May Aung, vol. $19, \mathrm{n}^{\circ} 23$, p. $32-37$.
Parasuraman, A. Valarie A. Zeithaml et Leonard L. Berry (1985), a A Conceptual Model of Service Quality and Its Implications for Future Research , Journal of Marketing, vol. $49, \pi^{\prime \prime} 2$, automne, p. 41-50.

Ryan, Chris, et Andrew Cliff (1997), w Do Travel Agencies Measure Up to Customer Expectations? An Empirical Investigation of Travel Agencies' Service Quality as Measured by SERVQUAL $\%$, Journal of Travel and Tourism Management, vol. 6, $\mathrm{n}^{\circ}$ 2, p. 1-31.

Québec, Tourisme Québec (1998), Pour donner au monde le goût du Québec : Politique de développement touristique.

Vallerand, André (1998), Le tourisme et le commerce électronique, in Exposés et lectures du IIT Colloque International de Management et Réseaux d'Entreprises, Université du Québec à Montréal, publies par Gilles St-Amant et Mokhtar Amami. 